\title{
Medical expenditure for esophageal cancer in China: a 10-year multicenter retrospective survey (2002-2011)
}

\author{
Lan-Wei Guo ${ }^{1,2+}$, Hui-Yao Huang ${ }^{2+}$, Ju-Fang Shi ${ }^{2}$, Li-Hong Lv³ ${ }^{3}$ Ya-Na Bai ${ }^{4}$, A-Yan Mao ${ }^{5}$, Xian-Zhen Liao ${ }^{6}$, \\ Guo-Xiang Liư ${ }^{7}$ Jian-Song Ren ${ }^{2}$, Xiao-Jie Sun ${ }^{8}$, Xin-Yu Zhu ${ }^{2,4}$, Jin-Yi Zhou ${ }^{9}$, Ji-Yong Gong ${ }^{10}$, Qi Zhou ${ }^{11}$, Lin Zhu ${ }^{12}$, \\ Yu-Qin Liu ${ }^{13}$, Bing-Bing Song ${ }^{14}$, Ling-Bin Du ${ }^{15}$, Xiao-Jing Xing ${ }^{16}$, Pei-An Lou ${ }^{17}$, Xiao-Hua Sun ${ }^{18}$, Xiao Qi ${ }^{19}$, \\ Shou-Ling Wu ${ }^{20}$, Rong Cao ${ }^{21}$, Li Lan ${ }^{22}$, Ying Ren ${ }^{23}$, Kai Zhang ${ }^{2}$, Jie He ${ }^{2}$, Jian-Gong Zhang ${ }^{1 *}$, Min Dai ${ }^{2^{*}}$ \\ and on behalf of the Health Economic Evaluation Working Group, Cancer Screening Program \\ in Urban China (CanSPUC)
}

\begin{abstract}
Background: Esophageal cancer is associated with substantial disease burden in China, and data on the economic burden are fundamental for setting priorities in cancer interventions. The medical expenditure for the diagnosis and treatment of esophageal cancer in China has not been fully quantified. This study aimed to examine the medical expenditure of Chinese patients with esophageal cancer and the associated trends.
\end{abstract}

Methods: From 2012 to 2014, a hospital-based multicenter retrospective survey was conducted in 37 hospitals in 13 provinces/municipalities across China as a part of the Cancer Screening Program of Urban China. For each esophageal cancer patient diagnosed between 2002 and 2011, clinical information and expense data were extracted by using structured questionnaires. All expense data were reported in Chinese Yuan (CNY; 1 CNY $=0.155$ USD) based on the 2011 value and inflated using the year-specific health care consumer price index for China.

Results: A total of 14,967 esophageal cancer patients were included in the analysis. It was estimated that the overall average expenditure per patient was 38,666 CNY, and an average annual increase of $6.27 \%$ was observed from 2002 $(25,111$ CNY) to 2011 (46,124 CNY). The average expenditures were 34,460 CNY for stage I, 39,302 CNY for stage II, 40,353 CNY for stage III, and 37,432 CNY for stage IV diseases $(P<0.01)$. The expenditure also differed by the therapy type, which was 38,492 CNY for surgery, 27,933 CNY for radiotherapy, and 27,805 CNY for chemotherapy $(P<0.05)$. Drugs contributed to $45.02 \%$ of the overall expenditure.

Conclusions: These conservative estimates suggested that medical expenditures for esophageal cancer in China substantially increased in the last 10 years, treatment for early-stage esophageal cancer costs less than that for advanced cases, and spending on drugs continued to account for a considerable proportion of the overall expenditure.

\footnotetext{
*Correspondence: jgzhang01@126.com; daimin2002@hotmail.com

†Lan-Wei Guo and Hui-Yao Huang contributed equally to this work

1 Department of Cancer Epidemiology, Henan Office for Cancer Control and Research, The Affiliated Cancer Hospital of Zhengzhou University, Henan Cancer Hospital, No. 127 Dongming Road, Zhengzhou 450008, Henan, P. R. China

2 Program Office for Cancer Screening in Urban China, Cancer Hospital, Chinese Academy of Medical Sciences (CAMS), Peking Union Medical College, National Cancer Center of China, No. 17 Panjiayuannanli, Chaoyang District, Beijing 100021, P. R. China

Full list of author information is available at the end of the article
} 
Keywords: Esophageal neoplasms, Medical expenditure, Diagnosis and treatment, China

\section{Background}

Esophageal cancer is the eighth most common cancer worldwide with estimates of 455,784 new cases and 400,156 deaths in 2012 [1]. The incidence varies in different physiographic regions, nations, and races. China had the highest number of new esophageal cancer cases (more than 223,000) worldwide in 2012 [2]. The number of new esophageal cancer cases in China will increase to 433,783 by the year 2035 [1]. In the meantime, in China, early detection of esophageal cancer has increased, while mortality has decreased $[3,4]$. As the survival for esophageal cancer patients is prolonging and the average cost of esophageal cancer treatments is increasing, the economic burden of patients has continued to grow. Therefore, the need for prevention strategies is urgent, and the implementation of effective measures should be based on evidence from health economic analyses.

The Cancer Screening Program in Urban China (CanSPUC), which was supported by the National Health and Family Planning Commission (NHFPC) in China, has been a crucial cancer prevention initiative that began in August 2012 [5]. The primary objective of the CanSPUC was to explore an appropriate implementation approach for screening of population at high risk and early diagnosis of major cancers (including lung, female breast, colorectal, esophageal, gastric, and liver cancers) in urban populations in China, promoting the use of mature screening and early diagnosis technology for common cancers to reduce mortalities. Taking funding and local conditions into account, the program covered 13 provinces/municipalities in China [including eastern (Beijing, Hebei, Liaoning, Jiangsu, Zhejiang, Shandong, and Guangdong), central (Heilongjiang, Henan, and Hunan), and western regions (Chongqing, Gansu, and Xinjiang)] [6] by 2014 in the first stage and targets urban residents who were between 40 and 69 years of age. As a part of the CanSPUC, the main content of the health economic evaluation includes expenditure and economic burden assessment, quality of life and health utility surveys, multi-angle project sustainability assessment, clinical diagnosis and treatment, and cost-effective model-based analysis. The goal is to provide the scientific evidence for expanding cancer screening and health decision-making in early detection and treatment programs for the Chinese population.

Few studies have examined the medical expenditure of Chinese patients with esophageal cancer and their associated trends [7]. In this study, we used data collected from a retrospective survey conducted by the CanSPUC to estimate the medical expenditures for esophageal cancer during the past 10 years, from 2002 to 2011, and to analyze the time trends over the long term.

\section{Methods \\ Data sources}

This survey was approved by the Ethics Committee of the Cancer Hospital, Chinese Academy of Medical Sciences (CAMS) and was conducted from 2012 to 2014 in 13 pilot provinces/municipalities in China. Basic information, clinical diagnosis, and medical expense information of patients with esophageal cancer (ICD10: C15), discharged between 2002 and 2011, were collected from historical medical records of 37 hospitals in these provinces/municipalities. Hospitals in China are organized according to a 3-tier system that recognizes a hospital's ability to provide medical care and medical education and conduct medical research [6]. Based on this system, hospitals are designated as primary, secondary, or tertiary institutions. Furthermore, based on the level of service provision, size, medical technology, medical equipment, management and medical care quality, these 3 grades are further subdivided into 3 subsidiary levels $\mathrm{A}, \mathrm{B}$, and $\mathrm{C}$, resulting in a total of 9 levels. In addition, one special level, $3 \mathrm{~A}$, is reserved for the most specialized hospitals. Among the information extracted, medical expense information, including the registration fee, ward bed fee, diagnosis fee, inspection fee, treatment fee, surgery fee, laboratory fee, nursing fee, drug fee, and other fees, refers to the medical costs for all physician visits from the first to the last treatments for esophageal cancer in the same hospital. This information was collected by a standard questionnaire or exported through the hospital electronic medical record (EMR) system, if possible. The database we created is a primary data source for this study that provides information on the diagnosis, treatment, and medical care costs for patients with esophageal cancer.

\section{Inclusion and exclusion criteria}

Esophageal cancer patients were enrolled in the study if they met the following criteria: (1) diagnosed with esophageal cancer (patients with advanced metastatic cancer should have esophageal cancer as the primary tumor); (2) previously hospitalized in one of the hospitals participating in the project, and the main treatment expenses occurred at that hospital; (3) basic personal and medical expense information was available, and clinical information (diagnosis, treatment, and pathologic diagnostics) 
was complete; and (4) the last hospital discharge was between Jan 1, 2002 and Dec 31, 2011.

The exclusion criteria were as follows: (1) diagnosed with two or more cancers; (2) not treated in the participating hospitals; (3) basic personal or medical expense information was not available, or the clinical information such as the time for the last discharge from the hospital, date of diagnosis, and overall medical expenditure was not complete; or (4) the last hospital discharge was not between Jan 1, 2002 and Dec 31, 2011.

\section{Sample size}

For each province/municipality, at least 120 esophageal cancer cases were selected in each year during 2002-2011, and the expected total sample size from 13 provinces/municipalities was 15,600 . In this survey, the proportion of eligible cases selected in each year in each city involved in the present survey was required to stay balanced for each clinical stage (I-IV) and gender, resulting in a sufficient number of cases for subgroup analysis.

\section{Quality control procedures}

First, the questionnaire design was confirmed by an expert reasoning committee. Second, esophageal cancer cases were selected according to the standard inclusion criteria. The information was collected via standard questionnaire by trained interviewers or exported through the hospital EMR system, if possible. Third, multi-level quality control was applied. The self-test was used when collecting information, and quality control staff then checked it again. Finally, a data administrator from the National Cancer Center of China would perform verification again after receiving the data. Data with mistakes or missing information was returned to the corresponding province/municipality.

\section{Statistical analysis}

A database was established using EpiData $v 3.1$ software (EpiData Association, Odense, Denmark). Data were entered at the provincial level; one person entered the data twice using the "Prepare Double Entry Verification" function in the EpiData $v 3.1$ software. SAS statistical software, version 9.3 (SAS Institute, Cary/NC, USA), was used for all statistical analysis. A two-tailed $P<0.05$ was considered statistically significant.

All expense data were reported in Chinese Yuan (CNY) based on the 2011 value, which was inflated using the year-specific personal health care consumer price index (CPI) of China. Medical expenditure per patient was defined as the total expenditure for all clinical visits per patient, and the reporting deadline for the expenses was the year corresponding to the last hospital discharge.
Medical expenditure per patient was described as the mean \pm standard deviation (SD) and compared using the 2 -sample Student $t$ test or ANOVA test after logarithm transition. The time trend of medical expenditures from 2002 to 2011 was described, and the Average Tempo was used to calculate an annual average increasing rate of medical expenditures.

\section{Results}

\section{Summary of the survey sites and hospitals}

Our study was conducted in 13 provinces/municipalities in China. The population size and gross domestic product (GDP) per capita in 2011 are presented in Table 1. Thirty-seven hospitals in 22 cities participated in the study, which included 22 general hospitals and 15 specialized hospitals.

\section{Characteristics of included esophageal cancer cases}

A total of 14,967 esophageal cancer patients (males accounted for $78.29 \%$ ), with an average diagnosis age of $60.93 \pm 9.90$ years (median: 61 years; percentile 25-percentile 75 [P25-P75]: 54-68 years), were included in the analysis (Table 2). Of these, $47.12 \%$ were from the eastern region of China, $77.08 \%$ were from specialized hospitals, and $91.88 \%$ were from 3A hospitals. Stage I esophageal cancer patients accounted for $7.56 \%$ of the sample, and esophageal squamous cell carcinoma (ESCC) was the most common pathologic type $(85.06 \%)$. The median total number of admissions/visits was 1 (percentile 5-percentile 95 [P5-P95]: 1-5), and the patients who only visited the hospital once accounted for $63.90 \%$ of the sample. The included patients spent a median of 28 hospitalization days receiving cancer treatment (range 1-507 days; P25-P75: 18-50 days). The main therapy types were surgery alone (34.75\%), surgery and chemotherapy (20.17\%), radiotherapy alone (13.33\%), and chemotherapy alone (11.36\%).

\section{Medical expenditure for esophageal cancer diagnosis and treatment per patient}

The average expenditure per patient increased with the clinical stage $(P<0.001)$. The expenditures also differed according to the therapy type, region, hospital level and category, number of clinical visits, gender, age at diagnosis, and pathologic type (all $P<0.05$ ). Additionally, since recent data are more applicable to current and future use, the expense data for the last 3 years (2009-2011) were also analyzed (Table 3 ). The average expenditures during 2009-2011 were higher than those during 2002-2011 for all subgroups.

The estimated overall average expenditure per patient was 38,666 CNY in 2002-2011 (Table 3), and more than 
Table 1 Summary of the survey sites and hospitals in 13 provinces/municipalities in China

\begin{tabular}{|c|c|c|c|c|c|c|}
\hline \multirow[t]{2}{*}{$\begin{array}{l}\text { Province/ } \\
\text { municipality }\end{array}$} & \multicolumn{2}{|c|}{$\begin{array}{l}\text { General information of the province/ } \\
\text { municipality }\end{array}$} & \multicolumn{4}{|c|}{ Specific information on survey sites and involved hospitals } \\
\hline & $\begin{array}{l}\text { Population } \\
\text { size in } 2011^{a} \\
(\times 10,000)\end{array}$ & $\begin{array}{l}\text { GDP per capita } \\
\text { in } 2011^{\mathrm{a}} \text { (CNY) }\end{array}$ & $\begin{array}{l}\text { No. of cities } \\
\text { involved }\end{array}$ & $\begin{array}{l}\text { No. of hospitals } \\
\text { involved }\end{array}$ & $\begin{array}{l}\text { No. (level) of } \\
\text { general hospitals } \\
\text { involved }^{\mathbf{b}}\end{array}$ & $\begin{array}{l}\text { No. (level) of spe- } \\
\text { cialized hospitals } \\
\text { involved }^{\text {b }}\end{array}$ \\
\hline \multicolumn{7}{|l|}{ Eastern region } \\
\hline Beijing & 2019 & 81,658 & 1 (Beijing) & 5 & $\begin{array}{l}3 \text { ( } 3 \mathrm{~A} \text { for } 2,3 \mathrm{~A} \text { less } \\
\quad \text { for } 1 \text { ) }\end{array}$ & 2 (3A for both) \\
\hline Hebei & 7241 & 33,969 & 1 (Tangshan) & 2 & 2 (3A for both) & 0 \\
\hline Liaoning & 4383 & 50,760 & $\begin{array}{l}2 \text { (Shenyang, } \\
\text { Tieling) }\end{array}$ & 2 & $1(3 \mathrm{~A})$ & $1(3 \mathrm{~A})$ \\
\hline Jiangsu & 7899 & 62,290 & $\begin{array}{l}2 \text { (Nantong, } \\
\text { Xuzhou) }\end{array}$ & 3 & 1 (3A less) & 2 (3A for both) \\
\hline Zhejiang & 5463 & 59,249 & $\begin{array}{l}2 \text { (Hangzhou, } \\
\text { Ningbo) }\end{array}$ & 2 & $1(3 A)$ & $1(3 \mathrm{~A})$ \\
\hline Shandong & 9637 & 47,335 & 1 (Jinan) & 1 & 0 & $1(3 A)$ \\
\hline Guangdong & 10,505 & 50,807 & $\begin{array}{l}5 \text { (Guangzhou, } \\
\text { Shenzhen, Dong- } \\
\text { guan, Foshan, and } \\
\text { Zhongshan) }\end{array}$ & 6 & 6 (3A for all) & 0 \\
\hline \multicolumn{7}{|l|}{ Central region } \\
\hline Heilongjiang & 3834 & 32,819 & 2 (Harbin, Daqing) & 4 & 2 (3A for both) & 2 (3A for both) \\
\hline Henan & 9388 & 28,661 & 1 (Zhengzhou) & 1 & 0 & $1(3 \mathrm{~A})$ \\
\hline Hunan & 6596 & 29,880 & 1 (Changsha) & 1 & 0 & $1(3 A)$ \\
\hline \multicolumn{7}{|l|}{ Western region } \\
\hline Chongqing & 2919 & 34,500 & 1 (Chongqing) & 1 & 0 & $1(3 A)$ \\
\hline Gansu & 2564 & 19,595 & $\begin{array}{l}2 \text { (Lanzhou, } \\
\text { Jinchang) }\end{array}$ & 8 & 6 (3A less for all) & $\begin{array}{l}2(3 \mathrm{~A} \text { for } 1,3 \mathrm{~A} \text { less } \\
\text { for } 1 \text { ) }\end{array}$ \\
\hline Xinjiang & 2209 & 30,087 & 1 (Urumqi) & 1 & 0 & $1(3 \mathrm{~A})$ \\
\hline Overall & - & - & 22 & 37 & 22 & 15 \\
\hline
\end{tabular}

a Based on the China Statistical Yearbook 2012, http://www.stats.gov.cn/tjsj/ndsj/2012/indexch.htm

b Hospitals in China are organized according to a 3-tier system that recognizes a hospital's ability to provide medical care and medical education and conduct medical research. Based on this, hospitals are designated as primary, secondary, or tertiary institutions. Furthermore, based on the level of service provision, size, medical technology, medical equipment, and management and medical care quality, these 3 grades are further subdivided into 3 subsidiary levels $A$, B, and C, resulting in a total of 9 levels. In addition, one special level, 3A, is reserved for the most specialized hospitals

90\% (36,173 CNY, 95\% confidence interval [CI]: 35,721$36,624 \mathrm{CNY}$ ) was spent within 2 months before and 10 months after the diagnosis.

The average expenditure per patient differed among geographical regions and survey sites. Of all 13 provinces/municipalities, Guangdong, Beijing, and Xinjiang had the highest expenditures (more than 50,000 CNY per patient); Hebei, Chongqing, and Gansu had the lowest expenditures (less than 30,000 CNY per patient); and Zhejiang, Heilongjiang, and Henan had nearly average expenditures (Fig. 1).

\section{Time trend of medical expenditures and related factors}

It was estimated that the overall average expenditure per patient increased by $6.27 \%$ per year from 25,111 CNY in 2002 to $46,124 \mathrm{CNY}$ in 2011. A turning point was observed around 2009 (Fig. 2a); the average rates of increase before and after that point were $8.60 \%$ and
$1.51 \%$, indicating that the average tempo slowed down after 2009. The expenditure per clinical visit increased by $2.15 \%$ per year from $20,117 \mathrm{CNY}$ in 2002 to 24,896 $\mathrm{CNY}$ in 2011 (Fig. 2b), and the daily average expenditure increased by $0.09 \%$ per year from $1262 \mathrm{CNY}$ in 2002 to $1274 \mathrm{CNY}$ in 2011 (Fig. 2c). The average number of clinical visits significantly increased from 1.25 in 2002 to 2.62 in 2011 (Fig. 2d). The length of hospitalization stay per patient significantly increased from 28.92 days in 2002 to 43.00 days in 2011 , and the longest stay was 45.00 days in 2008 (Fig. 2e).

The time trend of average expenditures differed according to geographical region, hospital type, hospital level, number of clinical visits, pathologic type, clinical stage, and therapy type (Fig. 3). The overall average expenditure per patient for the eastern and central regions of China increased faster than that for the western region of China, and the gap between those regions continued 
Table 2 Characteristics of the 14,967 selected esophageal cancer patients, 2002-2011

\begin{tabular}{|c|c|}
\hline Variable & Number of patients [cases (\%)] \\
\hline \multicolumn{2}{|l|}{ Region } \\
\hline Eastern & $7053(47.12)$ \\
\hline Central & $4287(28.64)$ \\
\hline Western & $3627(24.23)$ \\
\hline \multicolumn{2}{|l|}{ Hospital type } \\
\hline General & $3430(22.92)$ \\
\hline Specialized & $11,537(77.08)$ \\
\hline \multicolumn{2}{|l|}{ Hospital level } \\
\hline $3 \mathrm{~A}$ & $13,752(91.88)$ \\
\hline $3 \mathrm{~A}$ less & $1215(8.12)$ \\
\hline \multicolumn{2}{|l|}{ Gender } \\
\hline Male & $11,717(78.29)$ \\
\hline Female & $3250(21.71)$ \\
\hline \multicolumn{2}{|l|}{ Age at diagnosis (years) } \\
\hline$<45$ & $666(4.45)$ \\
\hline $45-54$ & $3252(21.73)$ \\
\hline $55-64$ & $5683(37.97)$ \\
\hline$\geq 65$ & $5366(35.85)$ \\
\hline \multicolumn{2}{|l|}{ Pathologic type ${ }^{a}$} \\
\hline Squamous cell carcinoma & $12,401(85.06)$ \\
\hline Others & $2178(14.94)$ \\
\hline \multicolumn{2}{|l|}{ Clinical stage } \\
\hline 1 & $1131(7.56)$ \\
\hline$\|$ & $5186(34.65)$ \\
\hline III & $4695(31.37)$ \\
\hline IV & $2880(19.24)$ \\
\hline Not reported & $1075(7.18)$ \\
\hline Morphologic verification & $13,332(89.10)$ \\
\hline \multicolumn{2}{|l|}{ Number of clinical visits per case } \\
\hline 1 & $9564(63.90)$ \\
\hline 2 & $2725(18.21)$ \\
\hline 3 & $1176(7.86)$ \\
\hline $4+$ & $1502(10.04)$ \\
\hline \multicolumn{2}{|l|}{ Type of therapy ${ }^{b}$} \\
\hline Surgery & $5168(34.75)$ \\
\hline Surgery and chemotherapy & $3000(20.17)$ \\
\hline Radiotherapy & $1982(13.33)$ \\
\hline Chemotherapy & $1690(11.36)$ \\
\hline Radiotherapy and chemotherapy & $1310(8.81)$ \\
\hline Surgery and radiotherapy & $386(2.60)$ \\
\hline Palliative care & $928(6.24)$ \\
\hline Others & $408(2.74)$ \\
\hline Comorbidities & $3877(25.9)$ \\
\hline Complications & $1235(8.25)$ \\
\hline
\end{tabular}

\footnotetext{
${ }^{a}$ The data for 388 patients were missing
}

b The data for 95 patients were missing to widen (Fig. 3a). The overall average expenditure per patient for the central region of China after 2008 was higher than that for the eastern region of China, and the gap gradually widened. The overall average expenditure per patient for the central region of China in 2011 was 1.73 times that for the western region of China. Additionally, the gap between general hospitals and specialized hospitals gradually decreased (Fig. $3 \mathrm{~b}$ ), but the gap between 3A hospitals and 3A less hospitals increased (Fig. 3c). The overall average expenditure per patient for 3A hospitals was 1.65 times that for 3A less hospitals in 2011. The overall average expenditure per patient for stage I disease was much lower than those for stages II, III, and IV diseases, and the gap increased after 2008 (Fig. 3g). The overall average expenditure per patient for stages II, III, and IV diseases did not show a significant difference. Stratification by therapy type showed that the overall average expenditure per patient increased faster for the chemotherapy alone, surgery alone, and surgery combined chemotherapy subgroups, but increased gradually for the palliative care subgroup (Fig. 3h). Further details on the expenditure time trends are also presented by the number of clinical visits, age at diagnosis, and pathologic type (Fig. 3).

\section{The proportional breakdown of medical expenditures}

Within the overall average expenditure, drug fee accounted for the largest proportion $(45.02 \%$, and never less than $40 \%$ since year 2003), followed by non-surgical treatment (19.25\%), surgery (10.10\%), inspection (5.69\%), laboratory test $(3.57 \%)$, and beds $(2.94 \%)$. The registration fee accounted for the smallest proportion (0.70\%). The proportion of drug fee significantly increased from $39.69 \%$ in 2002 to $44.00 \%$ in 2011, and reached the highest at $46.84 \%$ in 2004 . In contrast, the proportion of surgery fee dramatically decreased from $19.47 \%$ in 2002 to $7.12 \%$ in 2011 (Fig. 4).

\section{Discussion}

In the present study, we found that the overall average expenditure per patient for esophageal cancer increased by $6.27 \%$ per year from 2002 to 2011 , with an estimate of 38,666 CNY (95\% CI 38,178-39,155 CNY) during 20022011. Drugs accounted for the largest proportion of the overall expenditure per patient.

The first year after cancer diagnosis is a period with particularly high expenses and intensive treatment $[8,9]$. We found that more than $90 \%$ of the expenses occurred within 12 months, which was higher than China's GDP per capita $(36,018 \mathrm{CNY}$ in 2011) [10]. As a comparison, 
Table 3 Subgroup analysis of medical expenditures for esophageal cancer diagnosis and treatment per patient

\begin{tabular}{|c|c|c|c|c|c|c|}
\hline Variable & $\begin{array}{l}\text { Expenditure per patient } \\
\text { during 2002-2011 (CNY) }\end{array}$ & Statistics $^{\mathrm{a}}$ & $P$ & $\begin{array}{l}\text { Expenditure per patient } \\
\text { during } 2009-2011 \text { (CNY) }\end{array}$ & Statistics $^{a}$ & $P$ \\
\hline Overall & $38,666(38,178-39,155)$ & & & $45,319(44,425-46,213)$ & & \\
\hline Region & & 649.44 & $<0.001$ & & 306.1 & $<0.001$ \\
\hline Eastern & $41,943(41,202-42,684)$ & & & $47,213(46,021-48,406)$ & & \\
\hline Central & $40,410(39,646-41,173)$ & & & $52,765(51,100-54,430)$ & & \\
\hline Western & $30,234(29,200-31,269)$ & & & $32,555(30,585-34,526)$ & & \\
\hline Hospital type & & -6.75 & $<0.001$ & & -1.20 & 0.228 \\
\hline General hospital & $36,684(35,663-37,704)$ & & & $45,706(43,883-47,529)$ & & \\
\hline Specialized hospital & $39,256(38,700-39,812)$ & & & $45,182(44,157-46,207)$ & & \\
\hline Hospital level & & 25.60 & $<0.001$ & & 13.26 & $<0.001$ \\
\hline $3 \mathrm{~A}$ & $40,134(39,625-40,643)$ & & & $46,751(45,824-47,677)$ & & \\
\hline 3A less & $22,052(20,621-23,483)$ & & & $26,409(23,612-29,206)$ & & \\
\hline \multicolumn{2}{|c|}{ Number of clinical visits per patient } & 889.53 & $<0.001$ & & 204.28 & $<0.001$ \\
\hline 1 & $29,089(28,651-29,528)$ & & & $34,858(33,981-35,735)$ & & \\
\hline 2 & $46,633(45,530-47,737)$ & & & $53,959(52,002-55,917)$ & & \\
\hline 3 & $59,441(57,524-61,357)$ & & & $60,407(57,497-63,317)$ & & \\
\hline $4+$ & $68,928(66,774-71,082)$ & & & $69,055(65,749-72,360)$ & & \\
\hline \multicolumn{2}{|l|}{ Gender } & 5.64 & $<0.001$ & & 1.84 & 0.066 \\
\hline Male & $39,521(38,961-40,082)$ & & & $46,046(45,017-47,074)$ & & \\
\hline Female & $35,585(34,605-36,564)$ & & & $42,768(40,976-44,561)$ & & \\
\hline \multicolumn{2}{|l|}{ Age at diagnosis (years) } & 8.65 & $<0.001$ & & 14.42 & $<0.001$ \\
\hline$<45$ & $37,796(35,539-40,054)$ & & & $49,384(43,380-55,388)$ & & \\
\hline $45-54$ & $39,237(38,172-40,302)$ & & & $50,732(48,367-53,096)$ & & \\
\hline $55-64$ & $39,588(38,792-40,384)$ & & & $46,572(45,139-48,006)$ & & \\
\hline$\geq 65$ & $37,452(36,647-38,257)$ & & & $41,741(40,426-43,057)$ & & \\
\hline \multicolumn{2}{|l|}{ Pathologic type } & 23.95 & $<0.001$ & & 11.61 & $<0.001$ \\
\hline Squamous cell carcinoma & $40,711(40,173-41,250)$ & & & $48,242(47,233-49,250)$ & & \\
\hline Others & $30,216(29,032-31,399)$ & & & $35,576(33,636-37,516)$ & & \\
\hline \multicolumn{2}{|l|}{ Clinical stage } & 55.51 & $<0.001$ & & 21.80 & $<0.001$ \\
\hline I & $34,460(32,902-36,017)$ & & & $40,000(36,878-43,122)$ & & \\
\hline$\|$ & $39,302(38,526-40,079)$ & & & $47,978(46,381-49,575)$ & & \\
\hline III & 40,353 (39,491-41,214) & & & $44,962(43,616-46,309)$ & & \\
\hline IV & $37,432(36,180-38,685)$ & & & $42,972(40,749-45,194)$ & & \\
\hline \multicolumn{2}{|l|}{ Type of therapy } & 758.66 & $<0.001$ & & 270.53 & $<0.001$ \\
\hline Surgery & $38,492(37,890-39,094)$ & & & $47,778(46,548-49,008)$ & & \\
\hline Surgery and chemotherapy & $51,503(50,316-52,691)$ & & & $63,843(61,535-66,151)$ & & \\
\hline Radiotherapy & $27,933(26,787-29,079)$ & & & $29,085(27,471-30,699)$ & & \\
\hline Chemotherapy & $27,805(26,310-29,299)$ & & & $35,671(33,121-38,222)$ & & \\
\hline $\begin{array}{l}\text { Radiotherapy and chemo- } \\
\text { therapy }\end{array}$ & $53,907(51,867-55,946)$ & & & $57,234(53,865-60,603)$ & & \\
\hline Surgery and radiotherapy & $57,725(55,041-60,409)$ & & & $62,438(58,708-66,167)$ & & \\
\hline Palliative care & $13,846(12,626-15,067)$ & & & $15,627(13,187-18,067)$ & & \\
\hline Others & $23,057(20,564-25,550)$ & & & $21,821(18,386-25,256)$ & & \\
\hline
\end{tabular}

All expenditure data are presented as mean with $95 \%$ confidence interval in parentheses CNY Chinese Yuan

a The two-sample Student $t$ test after logarithm transition was used for binary classification variables, including hospital type, hospital level, gender, and pathologic type. The ANOVA test after logarithm transition was used for other multiple categorical variables, including region, number of clinical visits per patient, age at diagnosis, and clinical stage 


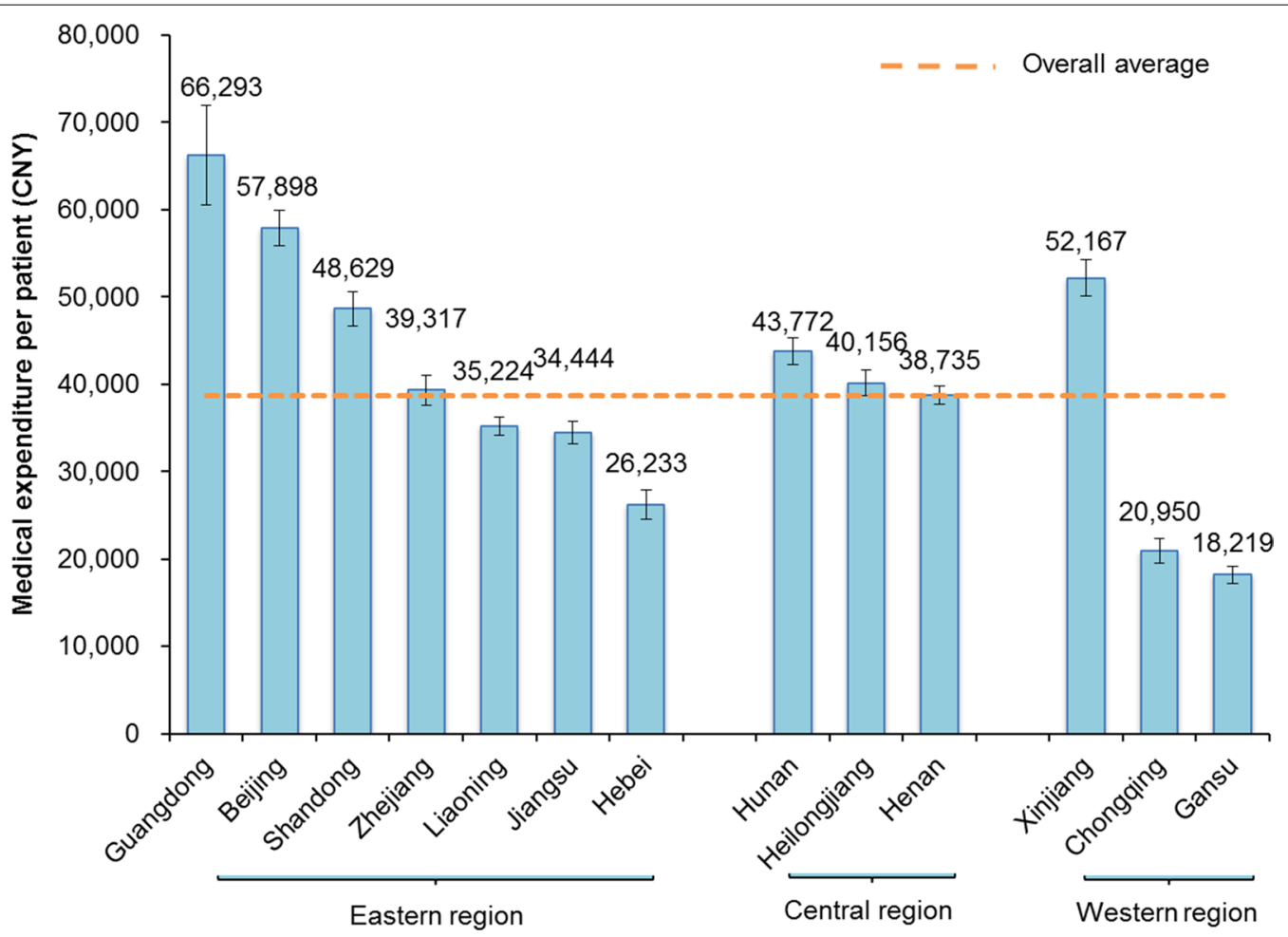

Fig. 1 Medical expenditure for esophageal cancer diagnosis and treatment per patient in China, by province/municipality. China is divided into the eastern, central, and western regions. Guangdong, Beijing, Shandong, Zhejiang, Liaoning, Jiangsu, and Hebei belong to the eastern region of China; Hunan, Heilongjiang, and Henan belong to the central region of China; and Xinjiang, Chongqing, and Gansu belong to the western region of China. Each bar indicates the average expenditure with 95\% confidence interval in parentheses

the newly diagnosed medical expenditure was less than $40 \%$ of the GDP per capita (2005) in the United Kingdom [11], less than 60\% (2008-2010) in the United States [12], and less than 65\% (1997-2007) in Canada [13]. According to data from China on the average family population (2.9) and the per capita disposable income $(21,810 \mathrm{CNY})$ of urban households in 2011 [10], we found that the average medical expenditure per patient for esophageal cancer was $72.9 \%$ of the average family annual income for urban households, indicating that the economic burden imposed by esophageal cancer was relatively high for individual patients' families in China, especially for lowincome families.

Our finding that the medical expenditures in the eastern and central regions were higher than those in the western region mirrors the heterogeneity in economic development among the three regions in China. However, the overall average expenditure per esophageal cancer patient in the Xinjiang province is an exception, which was estimated at 52,167 CNY. The economic burden of esophageal cancer in Xinjiang province is unusually high [6]. Further analysis indicated that esophageal cancer patients in Xinjiang province had more clinical visits than those in other provinces/municipalities. The medical expenditures per esophageal cancer patient significantly differed between the provinces/municipalities; there was a threefold gap between the highest (Guangdong in the eastern region) and lowest (Gansu in the western region) expenditure regions. This result was in agreement with other expenditure studies on esophageal cancer in China $[14,15]$.

Our finding of an expenditure increase of 1.84 times observed over 10 years is consistent with the results of some other studies. For example, Hu et al. [16] analyzed hospitalization expenses of 1819 patients with esophageal cancer in a cancer hospital in the Shanxi province and found that the adjusted average expenditure per patient increased from $8022 \mathrm{CNY}$ in 2002 to $33,723 \mathrm{CNY}$ in 2011. The time trend of expenditures was almost the same as the time trend of the length of hospitalization stay, suggesting that the length of stay was an important factor affecting the overall average expenditure per patient. Therefore, controlling the length of hospitalization stay has been considered an effective measure for reducing medical expenditures. For this reason, some hospitals try to shorten the hospitalization stays of esophageal cancer patients through implementing clinical pathways, promoting daytime chemotherapy, and 


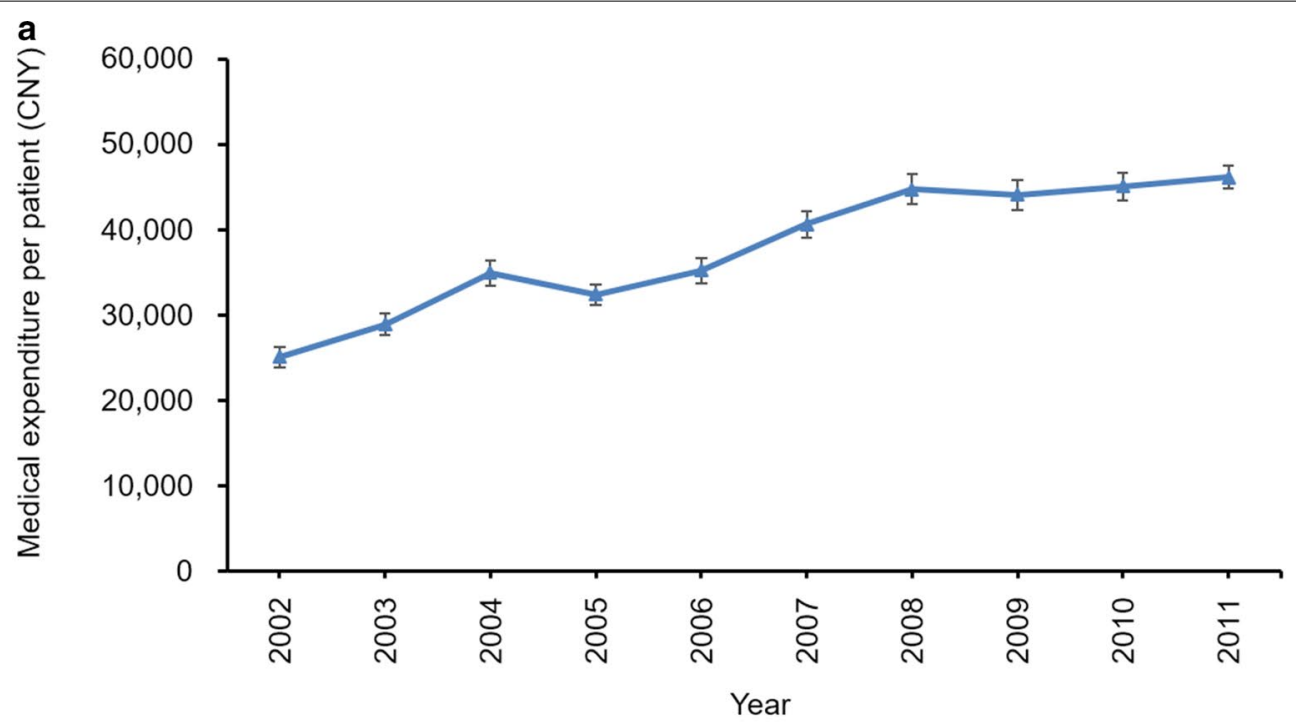

b
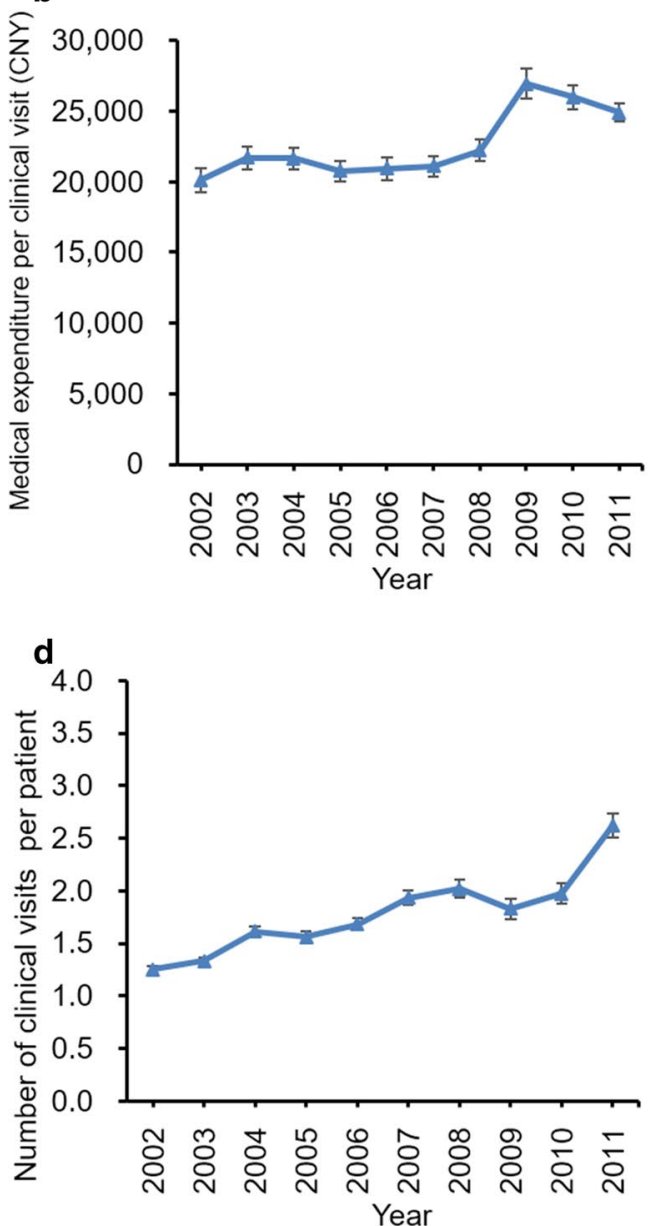

C
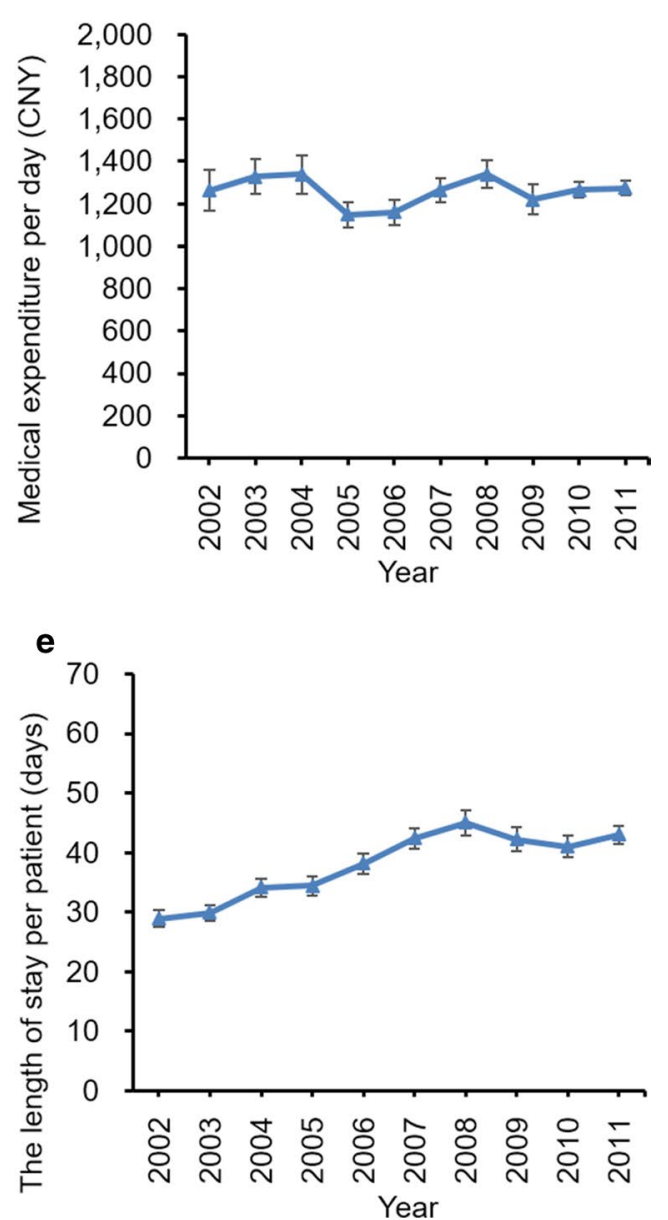

Fig. 2 Time trend of medical expenditures and related factors for esophageal cancer diagnosis and treatment in China, 2002-2011. a Time trend of overall average medical expenditures per patient; $\mathbf{b}$ time trend of medical expenditures per clinical visit; $\mathbf{c}$ : time trend of daily average medical expenditure; $\mathbf{d}$ time trend of number of clinical visits per patient; $\mathbf{e}$ time trend of the length of hospitalization stay per patient 


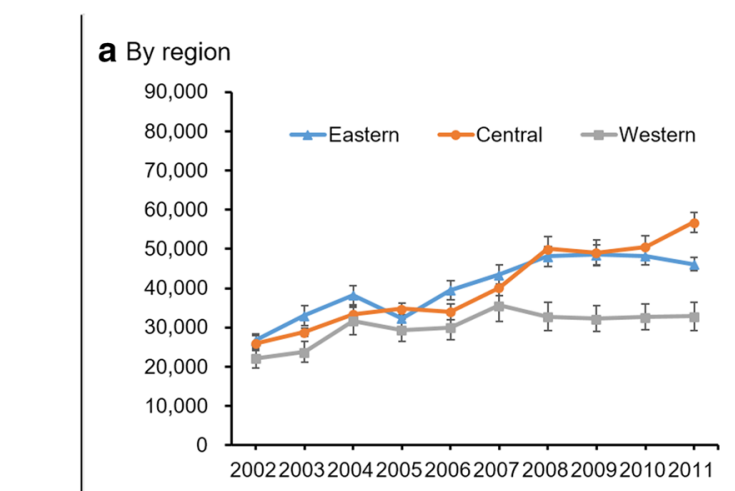

b By hospital type

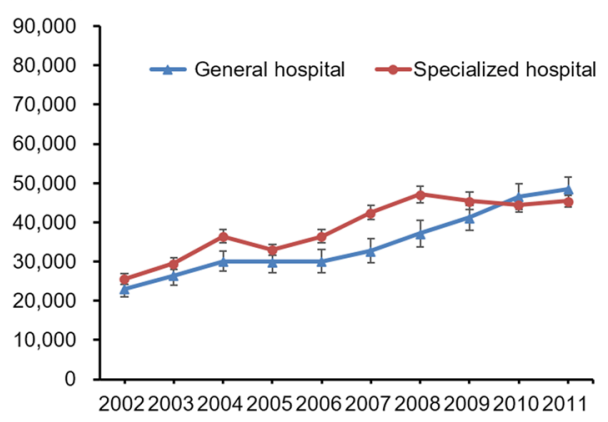

C By hospital level

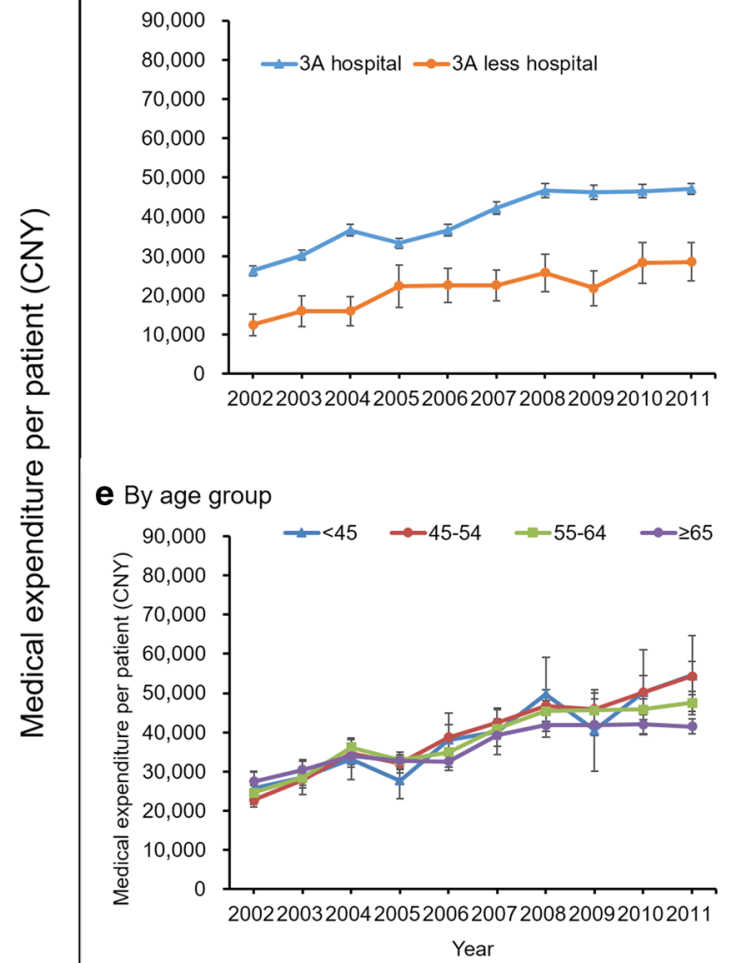

d By number of clinical visits

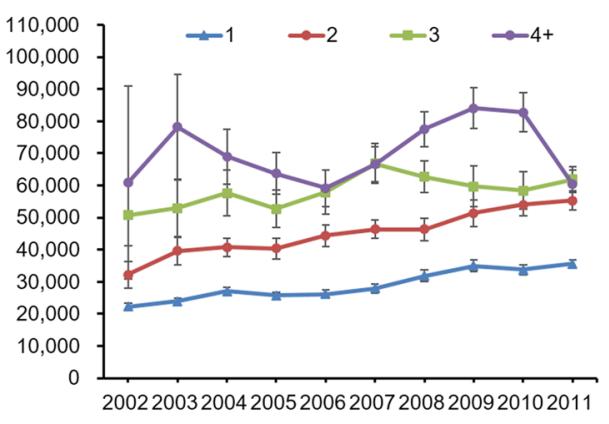

f By pathological type

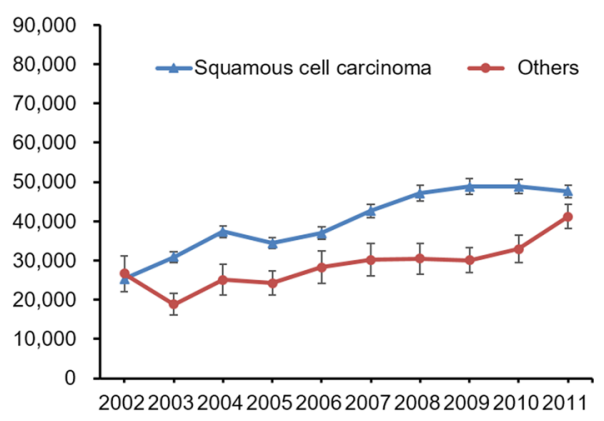

g By clinical stage

h By therapy type
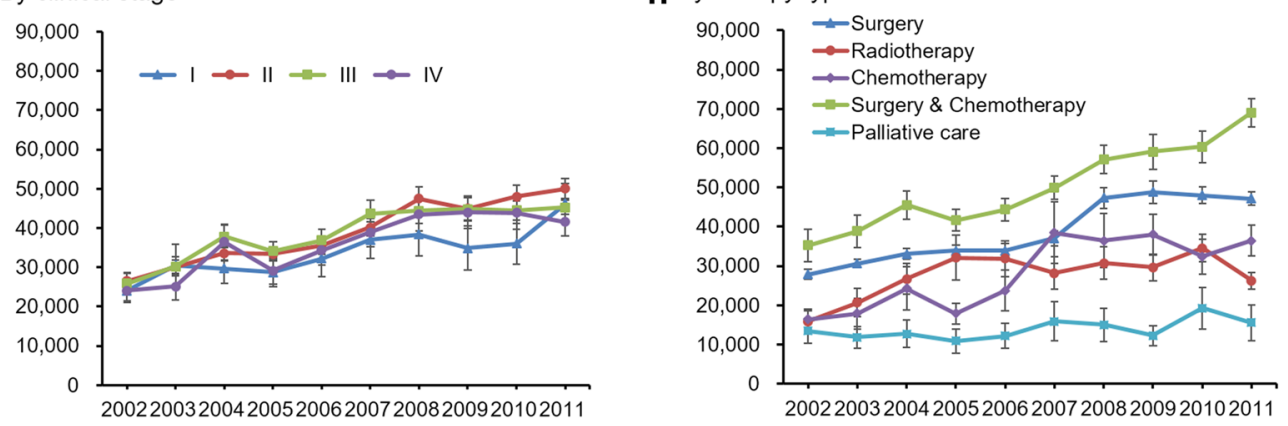
(See figure in previous page)

Fig. 3 Subgroup analysis on time trend of medical expenditures for esophageal cancer diagnosis and treatment per patient in China, $2002-2011$. a Time trend of medical expenditures stratified by region in China; $\mathbf{b}$ time trend of medical expenditures stratified by hospital type; $\mathbf{c}$ time trend of medical expenditures stratified by hospital level; $\mathbf{d}$ time trend of medical expenditures stratified by number of clinical visits per patient; $\mathbf{e}$ time trend of medical expenditures stratified by age group; $\mathbf{f}$ time trend of medical expenditures stratified by pathologic type; $\mathbf{g}$ time trend of medical expenditures stratified by clinical stage; $\mathbf{h}$ time trend of medical expenditures stratified by therapy type

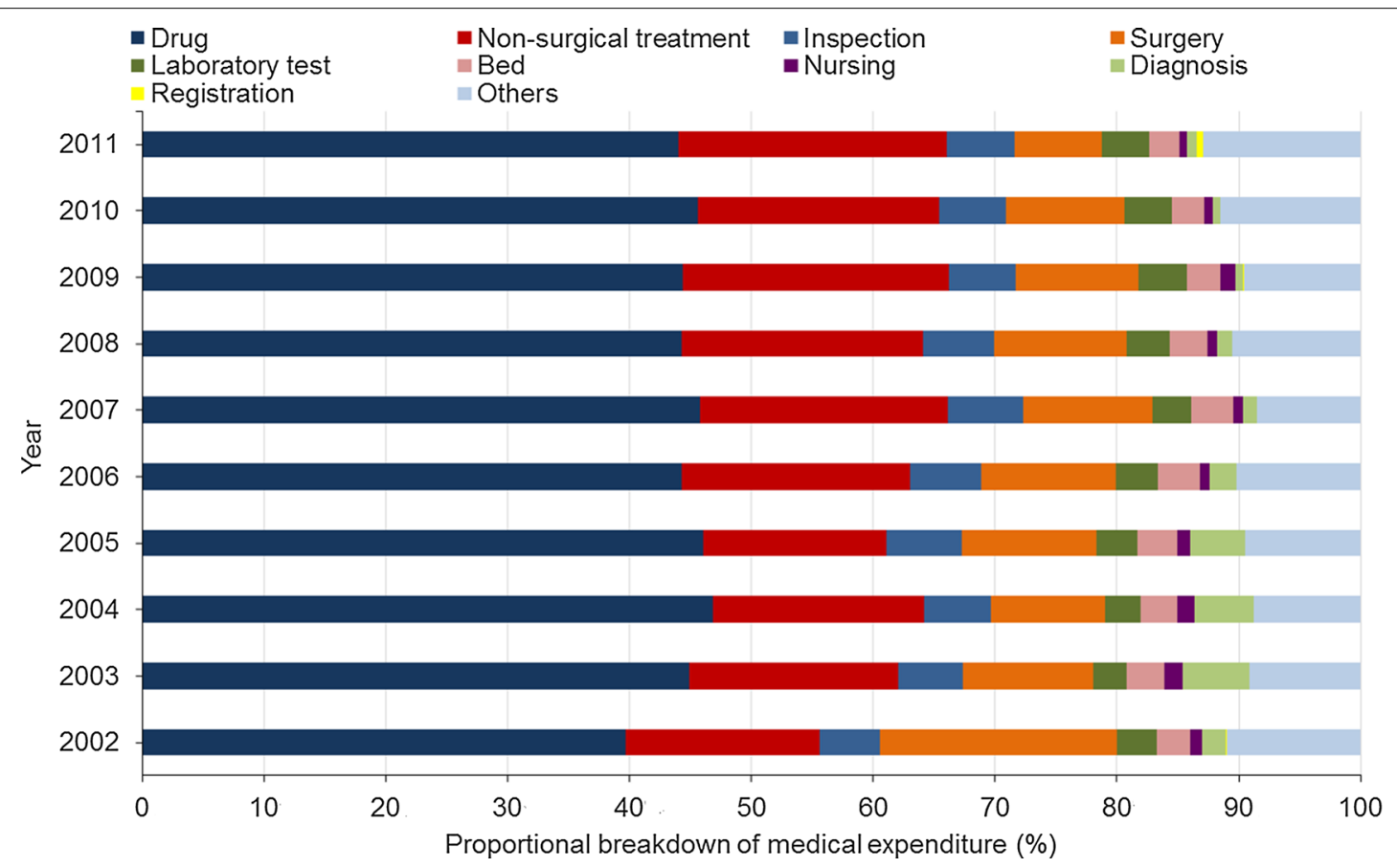

Fig. 4 The proportional breakdown of medical expenditures for esophageal cancer diagnosis and treatment per patient. Others include the expenditures for oxygen, blood infusion, heating, and so on

other approaches. The two peaks of medical expenditures observed in 2004 and 2008 in the present study might be attributed to the increase in clinical visits and hospitalization stay.

In the present study, we compared the time trend of medical expenditures for esophageal cancer among various subgroups. The expenditures were higher in specialized hospitals than in general hospitals and higher in 3A hospitals than in 3A less hospitals, which might be attributed to the more standardized diagnosis and treatment in specialized and 3A hospitals. The average expenditure per patient increased with the number of clinical visits. There were minor differences in the expenditures among age groups at diagnosis. Medical expenditures were lower for those diagnosed at 65 years and older than for younger patients. This was consistent with results from some other studies, such as a study in the United States that reported the overall expenditures among earlystage esophageal cancer patients older than 65 years of age are, on average, 20\% lower than those of younger patients [17]. This is probably because older patients have a smaller probability of cure, and physical or economic pressures forced the patients to abandon intensive treatment or to choose palliative care [18]. ESCC is the most common type of esophageal cancer in China [19]. The higher expenditure for ESCC than for other pathologic types was probably because of the availability of more therapy options and standardized procedures for ESCC. The average expenditure per esophageal cancer patient widely differed between therapy subgroups in the present study. The chemotherapy fee grew fastest, which was probably because of newly developed, expensive anticancer drugs. The expenditure of surgery were higher than those of chemotherapy or radiotherapy, which was probably because surgery is expensive and carries corresponding higher fees for diagnosis and inspection.

We found that the expenditure for stage II-III diseases was relatively higher than those for stage I and IV 
diseases, which seems to be reasonable if we take the differences of both survival and treatment among patients with I-IV diseases into consideration. As we all know, patients with stage I disease were mainly cured by surgery alone, which was relatively cheap, and those with stage IV disease was commonly treated with palliative care and with short survival period, whereas for patients with stage II and III diseases, multidisciplinary treatment were often adopted, which usually contain expensive antitumor drugs [20]. In addition, in the present study, the proportion of selected patients with stage I disease $(7.56 \%)$ was lower than the target proportion $(25 \%-$ $30 \%)$, even after substantial effort, indicating that most esophageal cancer patients were diagnosed with a late stage disease in the absence of routine esophageal cancer screening or early detection and treatment. A cancer screening program may help with the early diagnosis and treatment of esophageal cancer, which could decrease medical expenditure [21].

Our data showed that drug fee was the largest component of medical expenditure and had never been less than $40 \%$ since 2003, although China's health care reform (initiated in 2009) has planned to reduce the prices of drugs on essential drug lists [22]. The drug fee covers the cost of all the medicine used during the treatment, such as anticancer drugs and supportive drugs for symptoms, most of which are expensive joint venture or imported drugs. Additionally, esophageal cancer patients undergo multiple courses of chemotherapy, resulting in a high drug fee. Therefore, reducing drug fee is important for reducing the financial burden of esophageal cancer patients. In addition, the future policy on drug price addition and economic incentives in medical institutions may increase physician-induced demand, increasing the use of drugs and high-tech inspections [23]. In contrast, the proportions of diagnosis and nursing fees were very low, accounting for less than $3 \%$ of the overall medical expenditure, which also had a declining trend from 2002 to 2011. Overall, the expenditure proportion reflecting medical workers' labor value was still relatively low in the diagnosis and treatment of esophageal cancer. Adjusting charges for medical services to appropriately reflect the value of medical staff services, reduce physician-induced demand, and decrease medical expenditures is very important.

The current analysis has several limitations. First, the gender-specific and stage-specific sample sizes were controlled at the investigation phase, inducing selection bias and potentially decreasing the generalizability of the data. Second, this hospital-based survey only abstracted the expenditure data within the study hospitals; however, some patients also received diagnoses and treatments from other hospitals, suggesting that our data probably under-estimated the economic burden of esophageal cancer. Further follow-up studies are needed to overcome this limitation. Third, in this study, we did not obtain insurance reimbursement information, making it impossible to analyze the actual economic burdens from out-of-pocket payments among esophageal cancer patients during 2002-2011. Finally, this analysis only reported medical expenditure data and did not include non-medical expenditure, which is another important component of expenditure estimation from a patient's perspective. The non-medical expenditure will be separately reported by the health economic evaluation group of the CanSPUC program using data obtained from faceto-face interviews with esophageal cancer patients.

In conclusion, this study showed that medical expenditure for esophageal cancer in China was burdensome and substantially increased during 2002-2011. To dramatically decrease the economic burden on esophageal cancer patients, solutions are required to reduce personal payment ratio and to reduce medical expenditures incurred by services that are not covered by medical insurance. Our future analysis will use a model-based approach to perform a more aggressive estimate of the medical expenditure per esophageal cancer patient while considering variations, such as the numbers of clinical visits/admissions reported by individual study sites.

\section{Abbreviations \\ CanSPUC: Cancer Screening Program in Urban China; CAMS: Chinese Acad- emy of Medical Sciences; EMR: electronic medical record; CNY: Chinese Yuan; GDP: gross domestic product; CPI: consumer price index; ESCC: esophageal squamous cell carcinoma.}

\section{Authors' contributions}

LWG and JGZ led the data interpretation, analysis and manuscript drafting. JFS led the health economic study design for the CanSPUC program. $\mathrm{HYH}$ and $X Y Z$ contributed to data quality control and interpretation. LHL, YNB, AYM, $X Z L, G X L$, JSR and XJS contributed to data analysis planning. LHL, YNB, AYM, $X Z L, G X L$, JYZ, JYG, QZ, LZ, YQL, BBS, LBD, XJX, PAL, XHS, XQ, SLW, RC, LL and YR contributed to data collection for each survey center. MD, KZ and JH contributed to the overall design and co-management of the CanSPUC program. All authors reviewed and revised the manuscript. All authors read and approved the final manuscript.

\footnotetext{
Author details

${ }^{1}$ Department of Cancer Epidemiology, Henan Office for Cancer Control and Research, The Affiliated Cancer Hospital of Zhengzhou University, Henan Cancer Hospital, No. 127 Dongming Road, Zhengzhou 450008, Henan, P. R. China. ${ }^{2}$ Program Office for Cancer Screening in Urban China, Cancer Hospital, Chinese Academy of Medical Sciences (CAMS), Peking Union Medical College, National Cancer Center of China, No. 17 Panjiayuannanli, Chaoyang District, Beijing 100021, P. R. China. ${ }^{3}$ Department of Human Resources, The Affiliated Cancer Hospital of Zhengzhou University, Henan Cancer Hospital, Zhengzhou 450008, Henan, P. R. China. ${ }^{4}$ Institute of Epidemiology and Health Statistics, Lanzhou University, Lanzhou 730000, Gansu, P. R. China. ${ }^{5}$ Public Health Information Research Office, Institute of Medical Information, Chinese Academy of Medical Sciences (CAMS), Beijing 100020, P. R. China. ${ }^{6}$ Hunan Office for Cancer Control and Research, Hunan Provincial Cancer Hospital, Changsha 410006, Hunan, P. R. China. ${ }^{7}$ Department of Health Economics, School of Health Management, Harbin Medical University, Harbin 150081, Heilongjiang, P. R. China. ${ }^{8}$ Center for Health Management and Policy, Key Lab of Health Economics and Policy, Shandong University, Jinan 250012,
} 
Shandong, P. R. China. ${ }^{9}$ Institute of Chronic Non-Communicable Diseases Prevention and Control, Jiangsu Provincial Center for Disease Control and Prevention, Nanjing 210009, Jiangsu, P. R. China. ${ }^{10}$ Science and Education Department of Public Health Division, Shandong Tumor Hospital, Jinan 250117 , Shandong, P. R. China. ${ }^{11}$ Chongqing Office for Cancer Control and Research, Chongqing Cancer Hospital, Chongqing 400030, P. R. China. ${ }^{12}$ Teaching and Research Department, Affiliated Cancer Hospital of Xinjiang Medical University, Ürümqi 830011 , Xinjiang, P. R. China. ${ }^{13}$ Cancer Epidemiology Research Center, Gansu Provincial Cancer Hospital, Lanzhou 730050, Gansu, P. R. China. ${ }^{14}$ Heilongjiang Office for Cancer Control and Research, Affiliated Cancer Hospital of Harbin Medical University, Harbin 150081, Heilongjiang, P. R. China. ${ }^{15}$ Zhejiang Office for Cancer Control and Research, Zhejiang Cancer Hospital, Hangzhou 310022, Zhejiang, P. R. China. ${ }^{16}$ Liaoning Office for Cancer Control and Research, Liaoning Cancer Hospital \& Institute, Shenyang 110042, Liaoning, P. R. China. ${ }^{17}$ Department of Control and Prevention of Chronic Non-Communicable Diseases, Xuzhou Center for Disease Control and Prevention, Xuzhou 221006, Jiangsu, P. R. China. ${ }^{18}$ Ningbo Clinical Cancer Prevention Guidance Center, Ningbo NO. 2 Hospital, Ningbo 315010, Zhejiang, P. R. China. ${ }^{19}$ Department of Occupational Medicine, Tangshan People's Hospital, Tangshan 063001, Hebei, P. R. China. ${ }^{20}$ Health Department of Kailuan Group, Kailuan General Hospital, Tangshan 063000, Hebei, P. R. China. ${ }^{21}$ Department of Health Policy and Economic Research, Guangdong Provincial Institute of Public Health, Guangzhou 511430, Guangdong, P. R. China. ${ }^{22}$ Institute of Chronic Disease Prevention and Control, Harbin Center for Disease Control and Prevention, Harbin 150056, Heilongjiang, P. R. China. ${ }^{23}$ Urban Office of Cancer Early Detection and Treatment, Tieling Central Hospital, Tieling 112000, Liaoning, P. R. China.

\section{Acknowledgements}

This work was supported by the National Health and Family Plan Commission of P. R. China. The authors thank all members and staff of the Health Economic Evaluation Working Group of the Cancer Screening Program in Urban China (CanSPUC). The authors would also like to thank the expert panel of the CanSPUC program.

\section{Competing interests}

The authors declare that they have no competing interests.

\section{Availability of data and materials}

The datasets supporting the conclusions in this article will not be shared because of the data sensitivity and ethical restrictions imposed by the Chinese government.

\section{Ethical approval and consent}

This survey was approved by the ethics committee of the Cancer Hospital, Chinese Academy of Medical Sciences (CAMS).

Received: 18 October 2016 Accepted: 2 July 2017

Published online: 07 September 2017

\section{References}

1. Ferlay J, Soerjomataram I, Ervik M, Dikshit R, Eser S, Mathers C, et al. GLOBOCAN 2012 v1.0, cancer incidence and mortality worldwide: IARC CancerBase No. 11. Lyon Fr Int Agency Res Cancer. 2013. http://globocan. iarc.fr. Accessed 12 Feb 2016.

2. Chen WQ, Zheng RS, Zeng HM, Zhang SW. The incidence and mortality of major cancers in China, 2012. Chin J Cancer. 2016;35(1):73. doi:10.1186/ s40880-016-0137-8

3. Wei WQ, Yang J, Zhang SW, Chen WQ, Qiao YL. Analysis of the esophageal cancer mortality in 2004-2005 and its trends during last 30 years in China. Chin J Prevent Med. 2010;44(5):398-402. doi:10.3760/cma.j.i ssn.0253-9624.2010.05.007 (in Chinese).

4. Zhang SW, Zhang M, Li GL, Wei WQ, Meng FS, Liu ZC, et al. An analysis of incidence and mortality of esophageal cancer in China, 2003-2007. China Cancer. 2012;21(4):241-7 (in Chinese).
5. Dai M, Shi JF, Li N. The design and expected goal for cancer screening program in urban China. Chin J Prev Med. 2013;47(2):179-82 (in Chinese).

6. Ministry of Health of the People's Republic of China. China Health statistics yearbook 2012. Beijing: China Union Medical University Press; 2012. http://www.moh.gov.cn/htmlfiles/zwgkzt/ptjnj/year2012/index2012. html. Accessed 20 Mar 2017 (in Chinese).

7. Guo LW, Shi CL, Huang HY, Wang L, Yue XP, Liu SZ, et al. Economic burden of esophageal cancer in China from 1996 to 2015: a systematic review. Chin J Epidemiol. 2017;38(1):102-99. doi:10.3760/cma.j.i ssn.0254-6450.2017.01.020 (in Chinese).

8. Yabroff KR, Lamont EB, Mariotto A, Warren JL, Topor M, Meekins A, et al. Cost of care for elderly cancer patients in the United States. J Natl Cancer Inst. 2008;100(9):630-41. doi:10.1093/jnci/djn288.

9. Warren JL, Yabroff KR, Meekins A, Topor M, Lamont EB, Brown ML. Evaluation of trends in the cost of initial cancer treatment. J Natl Cancer Inst. 2008;100(12):888-97. doi:10.1093/jnci/djn175.

10. National Bureau of Statistics of China. China statistical yearbook 2012. Beijing: China Statistics Press; 2012. http://www.stats.gov.cn/tjsj/ndsj/2012/ indexch.htm. Accessed 20 Mar 2017 (in Chinese).

11. Agus AM, Kinnear H, O'Neill C, McDowell C, Crealey GE, Gavin A. Description and predictors of hospital costs of oesophageal cancer during the first year following diagnosis in Northern Ireland. Eur J Cancer Care (Engl). 2013;22(4):450-8. doi:10.1111/ecc.12046.

12. Guy GP Jr, Ekwueme DU, Yabroff KR, Dowling EC, Li C, Rodriguez JL, et al. Economic burden of cancer survivorship among adults in the United States. J Clin Oncol. 2013;31(30):3749-57. doi:10.1200/JCO.2013.49.1241.

13. de Oliveira C, Bremner KE, Pataky R, Gunraj N, Chan K, Peacock S, et al. Understanding the costs of cancer care before and after diagnosis for the 21 most common cancers in Ontario: a population-based descriptive study. CMAJ Open. 2013;1-8:2013. doi:10.9778/cmajo.20120013.

14. Yang J, Wei WQ, Niu J, He YT, Liu ZC, Song GH, et al. Estimating the costs of esophageal cancer screening, early diagnosis and treatment in three high risk areas in China. Asian Pac J Cancer Prev. 2011;12(5):1245-50.

15. Ma L, Chen XZ, Liu YQ, Hu XB, Gong HX, Bai YN. The direct economic burden analysis for four common cancer in elderly in Lanzhou city of China. Chin J Gerontol. 2011;31(17):3352-4. doi:10.3969/j.issn.10059202.2011.17.067 (in Chinese).

16. Hu ZP, Feng XX, Pan XF. Influence factors of hospitalization expense among esophageal cancer patients. Chin J Public Health. 2013;29(3):315-7 (in Chinese).

17. Mariotto AB, Yabroff KR, Shao Y, Feuer EJ, Brown ML. Projections of the cost of cancer care in the United States: 2010-2020. J Natl Cancer Inst. 2011;103(2):117-28. doi:10.1093/jnci/djq495.

18. Yang M, Xiao J, Chen DX, Gao YX, Lan SY, Zhuang X. Pathway analysis of influencing factors of the hospitalization expense of 8288 esophageal cancer patients. Modern Prevent Med. 2013;40(5):801-7 (in Chinese).

19. Guo LW, Zhang SK, Liu SZ, Chen Q, Zhang M, Quan PL, et al. Human papillomavirus type-18 prevalence in oesophageal cancer in the Chinese population: a meta-analysis. Epidemiol Infect. 2016;144(3):469-77. doi:10.1017/S0950268815001703.

20. Strik C, ten Broek RP, van der Kolk M, van Goor H, Bonenkamp JJ. Healthrelated quality of life and hospital costs following esophageal resection: a prospective cohort study. World J Surg Oncol. 2015;13:266. doi:10.1186/ s12957-015-0678-3.

21. Gao QY, Fang JY. Early esophageal cancer screening in China. Best Pract Res Clin Gastroenterol. 2015;29(6):885-93. doi:10.1016/j.bpg.2015.09.018.

22. Yip W, Hsiao W. Harnessing the privatisation of China's fragmented health-care delivery. Lancet. 2014;384(9945):805-18. doi:10.1016/ S0140-6736(14)61120-X.

23. Wagstaff A, Yip W, Lindelow M, Hsiao WC. China's health system and its reform: a review of recent studies. Health Econ. 2009;18(Suppl 2):S7-23. doi:10.1002/hec.1518. 